\title{
Transforming glaucoma care pathways: current glaucoma accreditation in UK optometry
}

(c) The Author(s), under exclusive licence to The Royal College of Ophthalmologists 2021

Eye (2022) 36:676-678; https://doi.org/10.1038/s41433-021-01820-7

The Royal College of Ophthalmologists' Way Forward project predicted the number of people in the UK with glaucoma would increase by $22 \%$ from 2015 to 2025 and by $44 \%$ from 2015 to 2035, while conceding this growth might underestimate demand [1]. The coronavirus pandemic has since thrown into stark relief the mismatch between capacity and demand, and the already long-established need for alternative pathways [2]. There is clearly a pressing need for out-patient transformation. One such option, given the potential workforce, is development of pathways involving primary care optometry beyond referral filtering. At the same time, ophthalmic services guidance from the Royal College of Ophthalmologists is set to provide the link between risk stratification in glaucoma and non-medical health care professional (HCP) accreditation levels required to undertake relevant roles in glaucoma pathways, including roles for optometrists.

The original NICE Glaucoma Guideline (CG 85) [3] set out recommendations linking requirements for training and accreditation with specific roles, defining three levels of 'permitted role' including: 'Monitoring' (but not treatment) of patients with ocular hypertension (OHT) or suspected Chronic Open Angle Glaucoma (COAG) with an established management plan; 'Detection and Diagnosis' of OHT and glaucoma suspect status; and 'Monitoring and Treatment' of patients with OHT, suspected COAG, and COAG; however, access to training and accreditation through the College of Optometrists' 3 levels of glaucoma higher qualifications has, arguably, remained limited. While publication of the Ophthalmic Common Clinical Competency Framework [4] aimed to support development of training and accreditation for HCPs from different professions in eye care delivery, including glaucoma [5], it is unclear to what extent this development has had impact within optometry. In terms of Independent Prescribing (IP), the NICE glaucoma update, NG81 (https://www.nice.org.uk/guidance/ng81/ evidence/full-guideline-pdf-4660991389), noted holding a 'nonmedical prescribing qualification alone (without a specialist qualification relevant to the case complexity of glaucoma being managed) is insufficient for managing glaucoma or related conditions.'

Data from the College of Optometrists indicates that 1340 optometrists had obtained the professional certificate in glaucoma, with $173(5.4 \%)$ and $28(2.1 \%)$ of these registrants having progressed to obtain the higher certificate and the 'new' diploma respectively. The previous 'old' diploma (offering exam-based accreditation without a formal training programme) had been achieved by 69 optometrists, giving a total diploma cohort of 97 , with $\sim 86 \%$ of these professionals (82 optometrists) also having the IP qualification. The distribution of those with the professional certificate, based upon 2020 post-code data, is shown in Fig. 1. Regional uptake data for the College's 3 levels of glaucoma higher qualification updated to May 2021 is summarised in Table 1.

These data on UK optometrists with higher qualifications in glaucoma merit consideration within the context of potential for transforming glaucoma care. While accepting that the GOS differs in Scotland [6], and that in England and elsewhere some patients at very low risk of developing glaucoma can be monitored by optometrists with core competencies (for example, monitoring patients with an IOP of $24 \mathrm{mmHg}$ or less or those previously discharged suspects), it is arguably the cohort of optometrists with the professional certificate who can offer capacity to the HES for cases currently reviewed, i.e., representative of a proportion of the case mix within HES backlogs. In terms of the highest level of autonomous practice, only 81 optometrists have obtained both the diploma in glaucoma and IP, and many in this cohort already work in glaucoma services, either full time (or predominantly so) within the HES. For example, 10\% of this cohort of 81 practitioners with both the diploma and IP work within glaucoma services at Manchester Royal Eye Hospital. Currently, it seems unlikely, nationally, that an autonomous primary care optometry service will be able to make a widespread contribution to backlogs without significant NHS investment in training for the profession, not least in view of clinical placements required to facilitate the higher certificate and diploma; however, there is an undoubtedly sizeable cohort of optometrists with the professional certificate, and with many more in training, some with funding from NHS England. From the data presented here, it would appear primary care optometry-based services for monitoring $\mathrm{OHT}$ and glaucoma suspect cases (with a clinical management plan) could be developed and implemented well beyond current levels known to exist in the community (https://www.locsu.co.uk/what-we-do/locsu-servicedirectory/). At the same time the importance of appropriate case selection within such services, and the significant workload required to formalise clinical management plans and to establish successful collaborative IT solutions ought not to be underestimated.

To our knowledge, there has not been any interrogation of data around glaucoma higher qualifications, exploring the distribution of the potentially available workforce within primary care. Although other models of care are being used to enhance capacity [2, 7], primary care is an important option; however, questions remain to refine our understanding of how some of the deficit in glaucoma capacity can be met through this workforce. The present data do not afford any analysis of workforce availability, rather the data presented show regional numbers, with some indication of location of registered professionals. The willingness of optometrists (and their employers) to

p-

rovide primary care services for glaucoma has not been quantified

Dedication: The authors would like to dedicate this editorial to the memory of their friend, colleague, and co-author Dr Paul Spry ( $7^{\text {th }}$ August 1971 - $26^{\text {th }}$ August 2021$)$.

Received: 16 September 2021 Revised: 27 September 2021 Accepted: 14 October 2021

Published online: 26 October 2021 


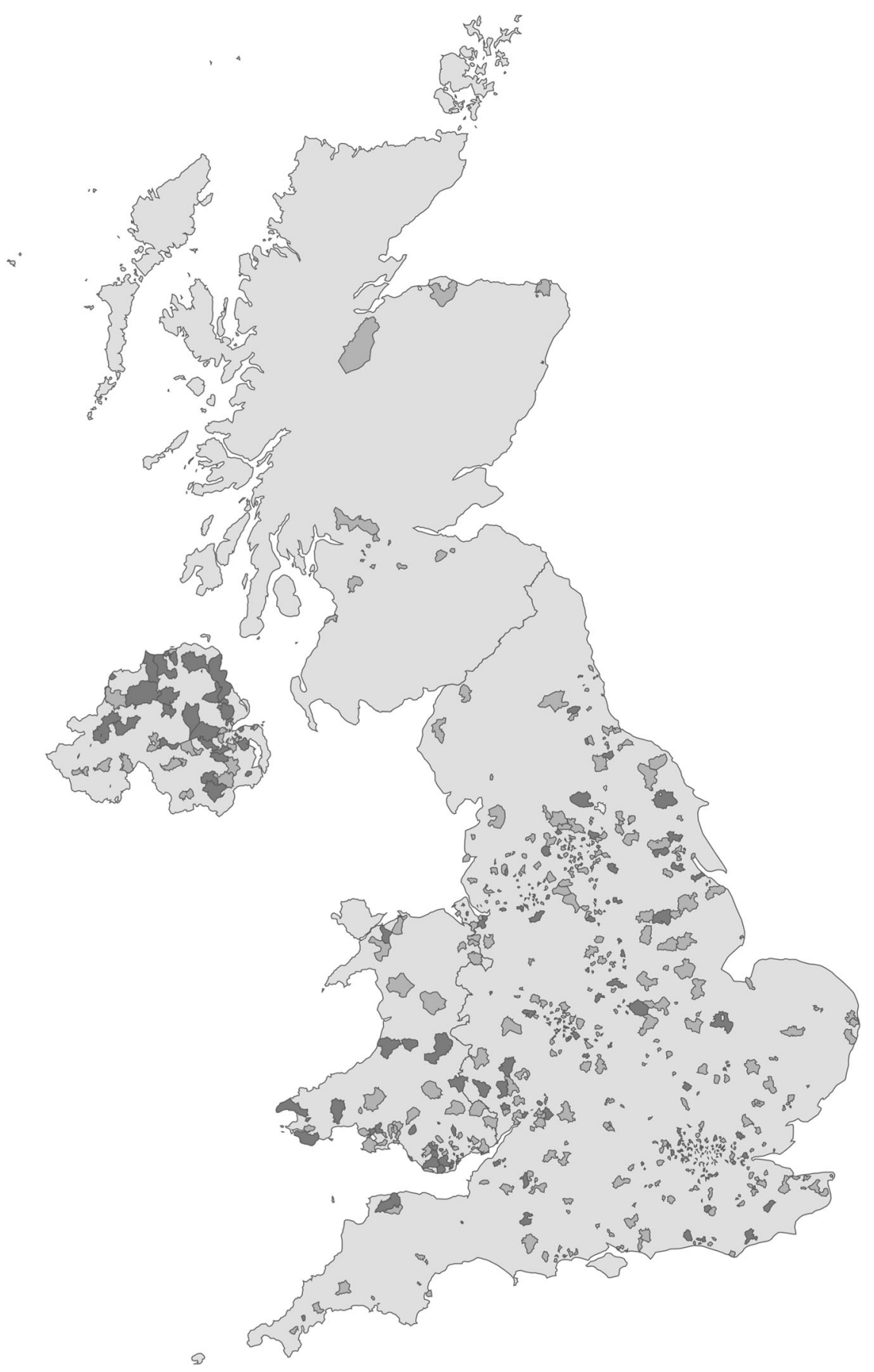

Fig. 1 Postcode based distribution of 1292 optometrists (2020 data) accredited with the College of Optometrists' Professional Certificate in Glaucoma. Post-codes in darker grey reflect greater numbers, but the figure does not provide granular representation of numbers per area (for a specific breakdown of regional numbers see Table 1); however, the figure does illustrate the relatively widespread distribution of holders of the professional certificate in England, Wales and Northern Ireland.

and is very likely to be impacted by the business model within the profession [8]. Furthermore, the current dataset does not distinguish between those already contributing to glaucoma pathways for monitoring, nor the provenance (i.e., primary versus secondary care) of those professionals currently engaged in glaucoma care.

An enhanced workforce evaluation allied to higher qualifications attained would help set the current context of capacity and demand and plans for transformation into a more strategic model, potentially requiring different approaches in different nations. Having knowledge of who has which qualifications, in which settings optometrists practise, and what their professional aspirations and willingness to further upskill might be, is important. Such data needs placing within a wider context, including funding for upskilling, current provision, national, regional, and local capacity, and projected demand. Data interrogation and further analyses should help clinical leads and commissioners better understand the primary care optometry workforce potential, in a scenario never more prescient than glaucoma services facing concerning backlogs. 
Table 1. College-accredited higher qualifications in glaucoma and Independent prescribing to May 2021.

\begin{tabular}{|c|c|c|c|c|}
\hline Region/Country & $\begin{array}{l}\text { Professional certificate in } \\
\text { glaucoma }\end{array}$ & $\begin{array}{l}\text { Higher certificate in } \\
\text { glaucoma }\end{array}$ & Diploma in glaucoma (new) & Independent prescribing \\
\hline Eastern & 74 & 16 & 3 & 39 \\
\hline North East & 28 & 4 & 1 & 36 \\
\hline North West & 90 & 18 & 4 & 96 \\
\hline West Midlands & 126 & 9 & 2 & 63 \\
\hline Yorks \& Humber & 194 & 21 & 1 & 57 \\
\hline England & 1034 & 152 & 22 & 593 \\
\hline Scotland & 22 & 2 & 1 & 362 \\
\hline Wales & 149 & 16 & 4 & 37 \\
\hline
\end{tabular}

Non-optometrists and those residing overseas have not been included. Data are organised by qualification category and by UK country/region within which registrants reside. Scotland has a much lower uptake of glaucoma higher qualifications (although proportionately a much higher uptake of IP), arguably a difference relating to GOS contract arrangements and optometric roles in Scotland compared to other parts of the UK, and to funding initiatives for IP. ${ }^{a}$ No regional data is available for the 'old' Diploma in Glaucoma, although these practitioners numbered 69, giving a UK total of 97 for the Diploma.

Robert A. Harper (iD ${ }^{1,2}$, Patrick J. G. Gunn (iD ${ }^{1,2}$, Paul G. D. Spry ${ }^{3}$, Cecilia H. Fenerty ${ }^{1,2,4}$, David P. Crabb ${ }^{5}$ and Michael Bowen ${ }^{6}$

${ }^{1}$ Manchester Royal Eye Hospital and Manchester Academic Health Sciences Centre, Manchester University NHS Foundation Trust Manchester, Manchester M13 9WL, UK. ${ }^{2}$ Division of Pharmacy and Optometry, School of Health Sciences, Faculty of Biology, Medicine and Health, University of Manchester, Manchester M13 9PL, UK. ${ }^{3}$ Bristol Eye Hospital, University Hospitals Bristol NHS Foundation Trust, Bristol BS1 2LX, UK. ${ }^{4}$ Division of Evolution and Genomic Sciences, School of Health Sciences, Faculty of Biology, Medicines and Health, University of Manchester, Manchester M13 9PL, UK. ${ }^{5}$ Division of Optometry and Visual Sciences, City, University of London, London EC1V OHB, UK. ${ }^{6}$ College of Optometrists, London WC2N 5NG, UK. 凶email: robert.harper@mft.nhs.uk

\section{REFERENCES}

1. The Way Forward: options to help meet demand for the current and future care of patients with eye disease: glaucoma. The Royal College of Ophthalmologists; London; 2017.

2. Harper RA, Gunn PJG, Fenerty CH, Spry PGD, Lawrenson JG. Care pathways for glaucoma detection and monitoring in the UK. Eye. 2020;34:89-102.

3. NICE. Glaucoma: diagnosis and management, clinical guideline [CG85]. 2009. https://www.nice.org.uk/guidance/CG85.

4. The common clinical competency framework for non-medical ophthalmic healthcare professionals in secondary care: glaucoma. RCOphth; London; 2016.

5. OCCCF online curricula. 2019. https://www.hee.nhs.uk/our-work/advanced-clinicalpractice/ophthalmology-common-clinical-competency-framework-curriculum.

6. Scottish Intercollegiate Guidelines Network (SIGN). Glaucoma referral and safe discharge. Edinburgh: SIGN; 2015. Guideline No. 144.

7. Ophthalmic services guidance: primary eye care, community ophthalmology and general ophthalmology. Royal College of Ophthalmologists and College of
Optometrists. 2019. https://www.rcophth.ac.uk/wp-content/uploads/2019/02/PrimaryEye-Care-Community-Ophthalmology-and-General-Ophthalmology-2019.pdf.

8. Sharma A, Jofre-Bonet M, Panca M, Lawrenson JG, Murdoch I. An economic comparison of hospital-based and community-based glaucoma clinics. Eye. 2012;26:967-971.

\section{AUTHOR CONTRIBUTIONS}

RAH, PJGG, PGDS and MB conceived this article, supported by CHF and DPC. MB collated the data with evaluation by RAH. RAH and MB wrote the manuscript and PJGG, CHF and DPC provided critical review of the manuscript.

\section{COMPETING INTERESTS}

MB is head of research at the College of Optometrists, where RAH is a council member, life fellow and examiner, and PJGG a member and examiner. The College of Optometrists is the provider of accreditation for those courses offering the glaucoma higher qualifications discussed in this article.

\section{ADDITIONAL INFORMATION}

Correspondence and requests for materials should be addressed to Robert A. Harper.

Reprints and permission information is available at http://www.nature.com/ reprints

Publisher's note Springer Nature remains neutral with regard to jurisdictional claims in published maps and institutional affiliations. 